\title{
Unusual B cell morphology in inflammatory bowel disease
}

\author{
Caterina Defendenti ${ }^{\mathrm{a}, *}$, Silvia Grosso ${ }^{\mathrm{a}}$, Fabiola Atzeni ${ }^{\mathrm{b}, \mathrm{c}}$, Annamaria Croce $^{\mathrm{d}}$, Olivia Senesi $^{\mathrm{d}}$, \\ Simone Saibeni ${ }^{\mathrm{e}}$, Simona Bollani ${ }^{\mathrm{e}}$, Piero Luigi Almasio ${ }^{\mathrm{f}}$, Savino Bruno ${ }^{\mathrm{e}}$, Piercarlo Sarzi-Puttini ${ }^{\mathrm{c}}$ \\ a Laboratory Unit, Fatebenefratelli Hospital, Milan, Italy \\ ${ }^{\mathrm{b}}$ Experimental Medicine, Queen Mary University, London, UK \\ ${ }^{\text {c }}$ Rheumatology Unit, L. Sacco University Hospital, Milan, Italy \\ d Division of Pathology, Fatebenefratelli Hospital, Milan, Italy \\ e Division of Gastroenterology, Fatebenefratelli Hospital, Milan, Italy \\ ${ }^{f}$ GI E Liver Unit, DI.BI.M.I.S., Policlinico, University of Palermo, Palermo, Italy
}

\section{A R T I C L E I N F O}

\section{Article history:}

Received 1 December 2011

Received in revised form 28 February 2012 Accepted 3 May 2012

\section{Keywords:}

Inflammatory bowel disease

Inflammation

Mucosal immunity

Lymphocytes

B-1 B cells

Lymphocyte homing

CD15+ cells

\begin{abstract}
A B S T R A C T
B lymphocytes express various different types of surface immunoglobulins that are largely unrelated to other hematological lines, although some reports have described a relationship between malignant B cells and other cells such as macrophages. Multiple genes of hematopoietic lineage, including transcription factors, are co-expressed in hematopoietic stem cells and progenitors, a phenomenon referred to as "lineage priming". Changes in the expression levels and timing of transcription factors can induce the lineage conversion of committed cells, which indicates that the regulation of transcription factors might be particularly critical for maintaining hierarchical hematopoietic development.

The aim of this study was to evaluate the surface markers of particular IgM-positive and irregularly nucleated cells detected in patients with inflammatory bowel disease (IBD), and to assess their association with diagnosis and inflammatory cell recruitment.

Small intestine, colon and rectal biopsy specimens of 96 IBD patients were studied. Immunoglobulinproducing cells (IPCs) were analyzed by means of immunofluorescence using polyclonal rabbit anti-human Ig and goat anti-human IgM. The specimens positive for B cells with irregular nuclei were assessed using monoclonal antibodies specific for CD79, and $\lambda$ and $\kappa$ chains in order to confirm their B cell nature. CD15+ cells, an important marker of inflammatory cell recruitment, were also evaluated. Statistical correlations were sought between the histological findings and clinical expression.

$34(35.4 \%)$ of the 96 patients (64 with ulcerative colitis and 32 with Crohn's disease) presented a periglandial localization of IPCs with irregular nuclei, which showed surface markers specific for the B cell subset, such as IgM and CD79, but quantitative differences in $\lambda$ and $\kappa$ chains. These specimens also contained CD15-positive cells, which are usually absent in healthy controls. The quantitative aspects and localization of the CD15-positive cells correlated with the distribution of the IPCs with irregular nuclei. IPCs with irregular nuclei were significantly more frequent in those patients with Crohn's disease than in those with ulcerative colitis $(p<0.001)$.

The finding of a subpopulation of cells that simultaneously showed irregular nuclei and B cell markers, such as functional surface IgM, in patients with IBD suggests that an unusual subgroup of B cells that correlates with CD15 expression and a diagnosis of Crohn's disease may be observed in the inflammatory process.
\end{abstract}

(c) 2012 Elsevier GmbH. All rights reserved.
Abbreviations: IBD, inflammatory bowel disease; IPC, immunoglobulinproducing cell; IFI, indirect immunofluorescence; $\mathrm{mAb}$, murine monoclonal antibodies; DAB 3, 3'-diaminobenzidine tetrahydrochloride; HRS, Reed-Sternberg cells; HL, Hodgkin lymphoma; HBV, Epstein-Barr virus; PC, phosphatidylcholine.

* Corresponding author at: Unità di Sierologia e Autoimmunità, Ospedale Fatebenefratelli, Corso di Porta Nuova 23, 20133 Milano, Italy. Tel.: +39 02 63632576; fax: +390263632348

E-mail address: caterina.defendenti@fbf.milano.it (C. Defendenti).

\section{Introduction}

B lymphocytes generally all have the same microscopic appearance [1] and express clonally diverse cell surface immunoglobulins. $B$ cells and other hematological lines are considered to be distantly related. In the literature is documented a relationship between malignant B cells and other cell types, such as macrophages, in contrast to the current paradigms. This aspect, known as "lineage infidelity", "lineage promiscuity", or "lineage switching", has not been investigated in depth because it has been attributed to 


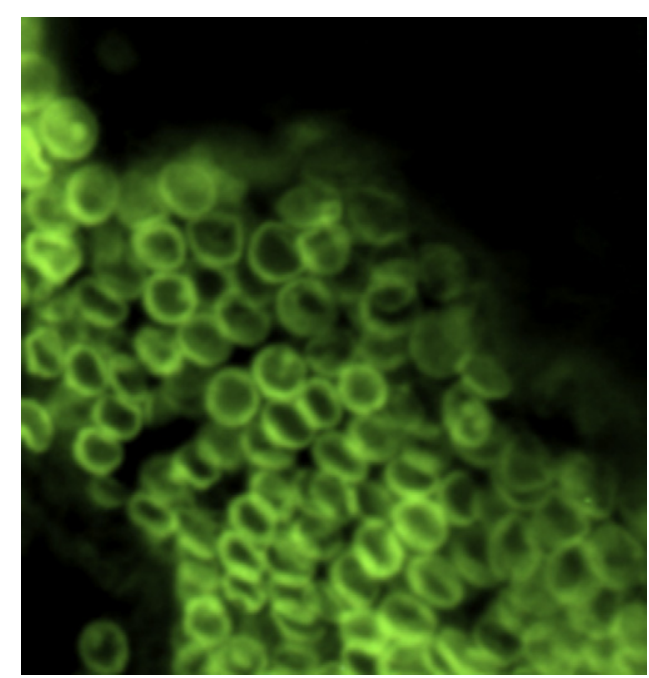

Fig. 1. Morphological appearance of IPCs on indirect immunofluorescence (magnification $40 \times$ ). These cells had a low cytoplasmic/nuclear ratio unlike conventional plasma cells. There is a fluorescent cut into the nucleus.

gene expression as a result of malignancy [2]. Despite numerous observations of B cell to macrophage transition in CD5+ B cell lymphomas, there is no previous data showing that normal $B$ cells could acquire this phenotype in vitro nor is there any evidence of $\mathrm{B} /$ monocytic cells in vivo. It has been found that monocytoid B lymphocytes are also associated with acquired immune deficiency syndrome (AIDS), chronic lymphocytic leukemia, Sjögren's syndrome and Hodgkin's disease [3]. Neoplastic Reed-Sternberg cells (HRS) have a monoclonal B cell nature, and single-cell analyses indicate a B lymphocyte origin based on the demonstration of clonal Ig gene rearrangements [4]. HRS cells attract various types of immune-system cells into lymphoma tissue, which leads to a typically inflammatory microenvironment [5].

However, a number of laboratories have demonstrated that, after co-culture with normal splenic fibroblasts or a fibroblastconditioned medium, a subset of normal mouse splenic B lymphocytes become B/macrophage cells [6]. Moreover, Borrello $[2,7]$ argues for the existence of normal biphenotypical cells with CD5+ B lymphocyte and macrophage characteristics, as has been demonstrated in mice. He considers normal $\mathrm{B} /$ macrophage cell function in an evolutionary context in which a primitive, flexible cell type may play a dual role in adaptive and innate immunity. B-1b cells can also undergo differentiation and acquire a mononuclear phagocyte phenotype upon attachment to substrate in vitro [2]. Re-cultivated floating B-1b cells adhere to plastic and differentiate into bipolar mononuclear cells and, during this differentiation, they spontaneously express both myeloid and lymphoid restricted transcription factors.

We have recently investigated the localization of immunoglobulin-producing cells (IPCs) in inflamed intestinal tissue samples from 96 patients with inflammatory bowel disease (IBD: 64 with ulcerative colitis [UC] and 32 with Crohn's disease (CD), and have found two different patterns of $B$ lymphocyte infiltrates: One $(42.7 \%$ of the cases) was characterized by the strong-to-moderate stromal localization of small $\operatorname{IgM}^{+} / \mathrm{CD}^{+} 9^{+} / \mathrm{CD}^{-} 0^{-} / \mathrm{CD}^{-} 1^{-} / \mathrm{CD}^{-} 3^{-} / \mathrm{CD}^{ \pm}$IPCs (Figs. 1 and 2 ) resembling $\mathrm{B} 1 \mathrm{~B}$ cells; the other $(57.3 \%)$ had no such detectable IPCs in stromal or epithelial tissues. The IPCs were significantly less frequent in patients with CD than in those with UC $(p=0.004)$ [8].

For the purposes of this study, we selected 34 tissue samples that showed IPCs with irregular nuclei in order to evaluate the surface markers of the particular IgM-positive and irregularly nucleated

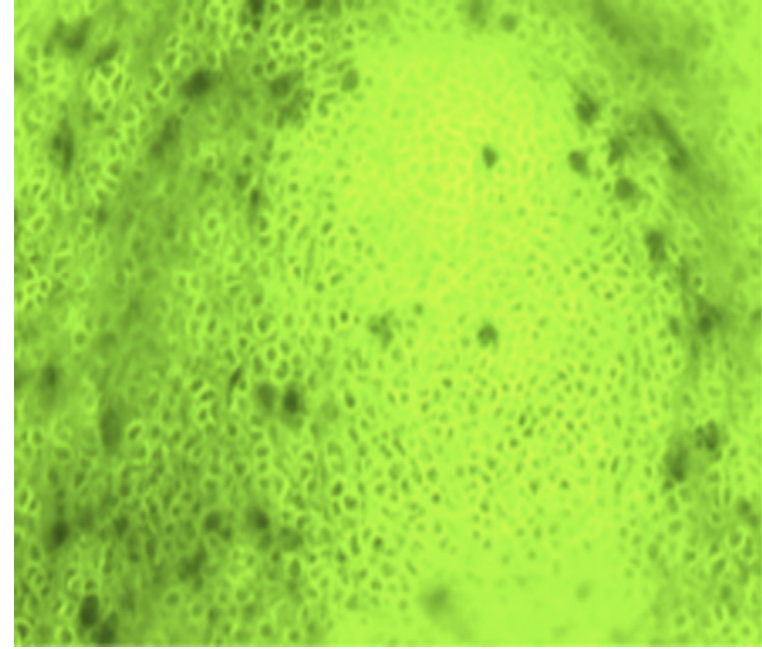

Fig. 2. Massive IPC invasion of intestinal tissue (magnification $20 \times$ ). In some biopsies, the presence of B-1 like cells was so important that the structure of intestinal tissue was unrecognizable.

cells observed in patients with IBD, and their association with inflammatory cell recruitment.

\section{Materials and methods}

\section{Patients}

Small intestinal, colonic and rectal tissue samples were obtained from 96 patients undergoing complete colonoscopy at Fatebenefratelli Hospital in Milan, Italy. These biopsy specimens were taken from inflamed mucosa, and for each biopsy three sections were analyzed. Informed consent was obtained from all of the patients before the procedure. In each case, the diagnosis was confirmed by means of standard endoscopic and histological criteria (the additional hematoxylin and eosin staining of each sample). Clinical data were obtained from patients' clinical records. Control specimens were also taken from ten patients (30 sections) with normal endoscopic findings and no macroscopic evidence of inflammatory or neoplastic disease. The biopsies were selected in such a way as to obtain information from all parts of the intestinal tract.

\section{Immunofluorescence analysis}

After deparaffinization $\left(72^{\circ} \mathrm{C}\right)$ and pre-treatment to enhance antigenicity $\left(95^{\circ} \mathrm{C} \times 36 \mathrm{~min}\right)$, we used indirect immunofluorescence (IFI) to detect the distribution of the tissue expression of immunoglobulins in intestinal sections $(3 \mu \mathrm{m})$ that had been then incubated for $30 \mathrm{~min}$ with horse serum at room temperature in order to prevent non-specific binding. After removing the blocking solution, the sections were incubated with a polyclonal rabbit antihuman Ig (Santa Cruz Biotechnology, Santa Cruz, CA, USA) or a goat anti-human $\operatorname{IgM}$ ( $\mu$ chain specific, Vector Laboratories, Burlingame, CA, USA). While being protected from direct light at $37^{\circ} \mathrm{C}$ for $1 \mathrm{~h}$, the samples were washed four times for $5 \mathrm{~min}$ in PBS High Salt (4 M, home made) and mounted. Images of each sample were taken from four randomly chosen fields (Euroimmun, Lubeck, Germany) at magnifications of $40 \times$ and $20 \times$.

\section{Immunoperoxidase analysis}

Murine monoclonal antibodies (mAbs) against CD79, CD15, $\lambda$ and $\kappa$ chains were purchased from Medical Systems (Tucson, AZ USA) and optimally diluted for use with Ventana detection kits and 
Table 1

Demographic and clinical characteristics of the patients in the study.

\begin{tabular}{lll}
\hline & Ulcerative colitis & Crohn's disease \\
\hline Patients & 64 & 32 \\
Sex-ratio M/F & $32 / 32$ & $21 / 11$ \\
Age at diagnosis (mean, range) & $44.2 \pm 14.2$ & $47.2 \pm 16.6$ \\
Free of therapy at diagnosis & $18(28.1 \%)$ & $16(50.0 \%)$ \\
Surgical therapy & $3(4.7 \%)$ & $10(32.3 \%)$ \\
Disease severity & & \\
$\quad$ Inactive & $27(42.2 \%)$ & $11(34.4 \%)$ \\
$\quad$ Mild & $19(29.7 \%)$ & $15(46.9 \%)$ \\
$\quad$ Moderate & $14(21.9 \%)$ & $2(6.3 \%)$ \\
$\quad$ Severe & $4(6.3 \%)$ & $4(12.5 \%)$ \\
\hline
\end{tabular}

automated slide stainers (Medical Systems) before being applied to formalin-fixed, paraffin-embedded tissue. Biotinylated secondary antibodies were then added, followed by streptavidin-horseradish peroxidase conjugate. Each step in the staining protocol included incubation for a precise time at a specific temperature, after which the sections were rinsed by the Ventana automated slide stainer to stop the reaction and remove any unbound material that would hinder the desired reaction in subsequent steps. A coverslip solution was used in the slide stainer in order to minimize the evaporation of the aqueous reagents from the specimen-containing slide. The complex was then visualized using a hydrogen peroxide substrate and 3,3'-diaminobenzidine tetrahydrochloride (DAB) chromogen.

\section{Clinical score}

The patients were diagnosed as having CD or UC by means of standard criteria (i.e., endoscopy, histology, barium contrast enema, or other recognized criteria). Disease activity was defined on the basis of the Crohn's Disease Activity Index (CDAI) and biological markers of inflammation; in the case of UC, we used the Disease Activity Index (DAI)) validated by the St Mark's Hospital school [8-10].

\section{Statistical analysis}

The continuous variables were recorded as mean values and standard deviations and were compared using an unpaired Student's t-test. The categorical variables were recorded as absolute and relative frequencies and compared using the Chi-squared test. The data were analyzed using the Statistical Package for Social Sciences (SPSS 13.0; SPSS Inc., Chicago, IL, USA). All of the tests were two-sided and $p$ values of $<0.05$ were considered statistically significant.

\section{Results}

\section{Study population}

The study population consisted of 96 patients (64 with UC and 32 with $C D$ ), the majority of whom were males. Their demographic and clinical characteristics are shown in Table 1.

\section{IFI analysis distinguishes IPCs with irregular nuclei}

The analysis of IPCs in the biopsy specimens allowed the identification of cells with irregular nuclei (Figs. 3 and 4) which, as they were not detected in ten sections of normal intestinal tissue, do not seem to form part of the physiological intestinal cell population. They were characteristically distributed in the periglandial zone, had a sporadic distribution and never contributed to massive tissue infiltrates. These cells were found in 34 patients (35.4\%). We also observed small subepithelial IPCs, characterized by a large central

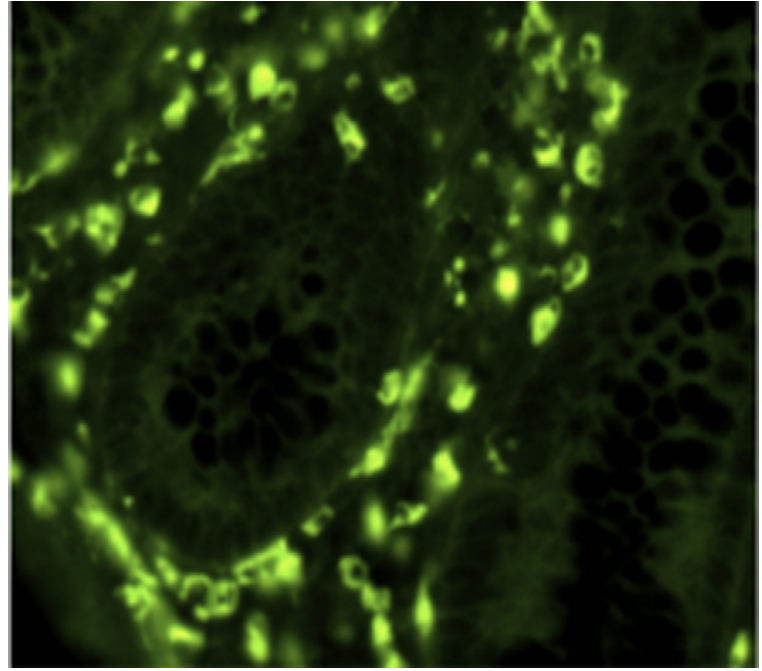

Fig. 3. IPC cells with irregular nuclei on intestinal tissue (magnification $40 \times$ ). These cells had a periglandial distribution and were characterized by the positivity of the chain $\mu$ on immunofluorescence in spite of the irregular aspect of their nucleus.

nucleus, distributed within the glands of 41 other IBD patients. These small IPCs, which were surface IgM positive, were set against IPCs with irregular nuclei because they were monomorphic, and often massively infiltrated inflamed tissues. Morphologically, they had a low cytoplasmic/nuclear ratio, unlike conventional plasma cells, and they were also present as discrete foci on ten sections of normal intestinal tissue, thereby seemingly contributing to the physiological intestinal cell population.

\section{$B$ cell surface phenotype analysis of inflammatory infiltrates identifies a distinct $C D 79+/ \lambda / \kappa$ chain + subset of IPCS}

The B cell-specific surface molecules IgM and CD79 were examined in the 34 samples showing an infiltrate of IPCs with irregular nuclei, all of which were positive for CD79 and $\kappa / \lambda$ chains. These data were confirmed by immunofluorescence and showed that surface Ig expression can be used as a marker to characterize B cells. Surprisingly, $\lambda$ chains were more represented than $\kappa$ chains.

The finding of IPCs within the intestinal specimens was statistically associated with a diagnosis of $\mathrm{CD}(p<0.001)$ (Table 2$)$.

Treatment did not influence the expression of different type of cells (Table 3 ) as $\mathrm{CD}^{+} 9^{+} / \mathrm{CD} 20^{-} / \mathrm{CD} 21^{-} / \mathrm{CD} 23^{-} / \mathrm{CD}^{ \pm}$IPC were observed with similar frequency in both treated and untreated

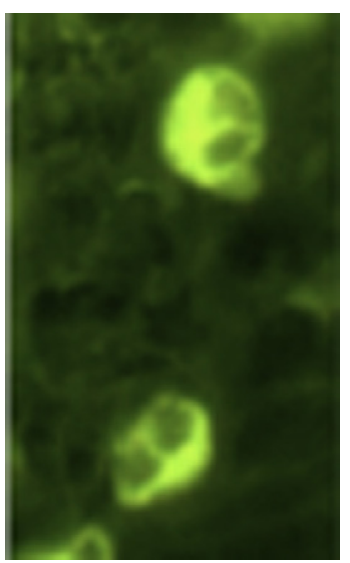

Fig. 4. IPC cells with irregular nuclei (detail). The unusual morphology of these B cells depends, in particular, on the aspect of their nucleus. 
Table 2

The finding of IPCs within the intestinal specimens and association with a diagnosis of $\mathrm{CD}$.

\begin{tabular}{llll}
\hline B cells & $\begin{array}{l}\text { Crohn's disease } \\
(n=32)\end{array}$ & $\begin{array}{l}\text { Ulcerative colitis } \\
(n=64)\end{array}$ & $p$ \\
\hline B-1 cells like & $7(21.9 \%)$ & $34(54.1 \%)$ & $<0.001$ \\
Irregular nuclei cells & $21(65.6 \%)$ & $13(20.3 \%)$ & \\
Other morphologies & $4(12.5 \%)$ & $17(26.6 \%)$ & \\
\hline
\end{tabular}

Table 3

Association between treatment and the expression of different type of cells.

\begin{tabular}{llll}
\hline B-1 cells like & Therapy & Therapy free & $p$ \\
\hline All patients & $29 / 62(46.8 \%)$ & $12 / 34(35.3 \%)$ & 0.3 \\
RU & $4 / 16(25.0 \%)$ & $4 / 12(18.8 \%)$ & 0.7 \\
CD & $26 / 46(56.5 \%)$ & $8 / 18(44.4 \%)$ & 0.4 \\
\hline
\end{tabular}

patients, and there was no significant difference between the IBD subgroups (Table 3 ).

\section{CD15+ cell distribution}

To evaluate the distribution of inflammation in patients with IPCs irregular nuclei, the 96 IBD patients in the study and the 10 healthy samples were tested for CD15+ cells. All of the healthy samples were negative, but all of the 34 IBD samples containing IgM+ ICPs with irregular nuclei showed a substantial infiltration of CD15+ cells, mainly in the periglandial zone (Fig. 5). In the remaining 62 samples, the distribution of the CD15 marker CD15 was variable without a well-defined correlation with the morphology and number of IPCs. In particular, some of the samples showed a considerable interstitial infiltration of CD15+ cells, with or without periglandial involvement (Fig. 6). The remaining samples were negative for CD15+ cells in both the interstitium and the periglandial zone, but expressed CD15+ in the crypts of the intestinal epithelium (Fig. 7)

\section{Discussion}

In this study, we identified IgM+ IPCs with irregular nuclei in intestinal tissue specimens taken from 34 patients with IBD, but in none of the samples taken from healthy subjects. The cells expressed B cell surface markers (CD79 and IgM), but showed quantitative differences in $\kappa / \lambda$ chains. They had a periglandial

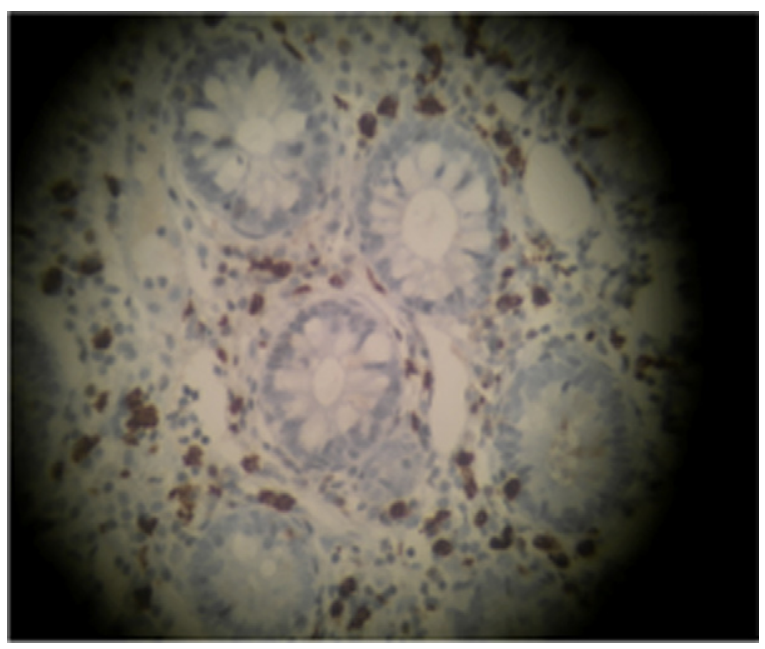

Fig. 5. CD15-positive cells within the periglandial zone of intestinal tissue. In 34 patients, those cells that were CD15 positive had the same distribution as the B cells with irregular nuclei found on imnunofluorescence.

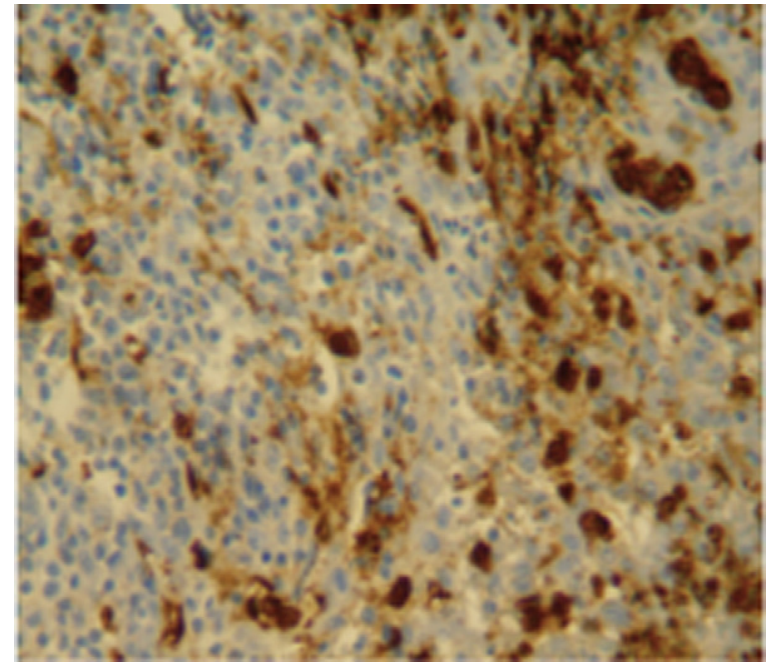

Fig. 6. CD15-positive cells within the interstitium of intestinal tissue. In a subgroup of patients negative for IPCs with irregular nuclei, CD15 positive inflammatory cells were present not only in the periglandial area but also in the circostante interstitium, and these were sometimes associated with necrosis due to enzymes released by granulocytes.

distribution. The same periglandial distribution also involved CD15 immunoperoxidase-positive cells, the neutrophil and/or lymphocyte/monocyte origin of which is difficult to define. These histological data are significantly associated with a diagnosis of CD.

Evaluation of the 62 patients negative for IgM+ ICPs with irregular nuclei revealed a different topographic distribution of the CD15 marker; it was found either only in the crypts of the intestinal epithelium [9] or in both the periglandial zone and the interstitium, and was sometimes associated with necrosis caused by enzymes generated by granulocytes.

$\mathrm{CD} 15$ recognizes the sugar moiety, lacto-N-fucopentaose, which is linked to cell membrane protein in various cells, but not normal B cells. The flow cytometric detection of the CD15 antigen is a marker of granulocytes, macrophages and activated T cells [10], and the antigen is also found in 37-100\% of Hodgkin/Reed-Sternberg cells (which are ICPs), and the malignant cells of Hodgkin lymphoma (HL).

The intestinal inflammatory process is characterized by the up-regulation of E-selectin and the recruitment of CD15+ cells. In patients with UC, CD15 expression detected by means of

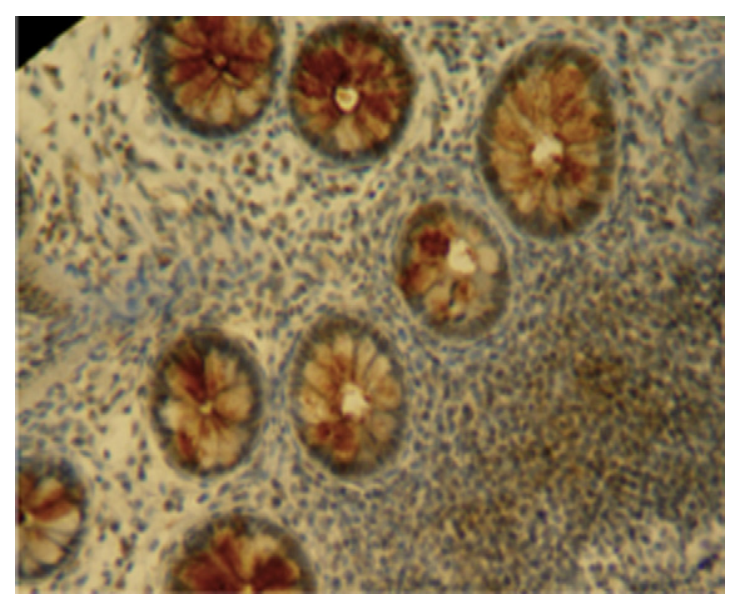

Fig. 7. CD15-positive cells in the crypts of the intestinal epithelium. On re-imaging patients' CD15 was not observed in the inflamed areas but rather in the normal histological component of the instestinal mucosa. 
immunoperoxidase correlates with histological scores, endoscopic severity and clinical activity. But no direct correlation with the effector cell subtype in gut mucosal inflammation has been demonstrated [11]. There are usually abundant, unique glycan structures in normal descending colon mucin. The functional implication of this variation is that different mucins may be recognized by different carbohydrate-binding proteins, such as bacterial adhesins and endogenous lectins (e.g. CD15), and may therefore play different specific roles in host-pathogen interactions, inflammation and cancer [12].

The limited distribution of CD15+ cells in the periglandial zone of the tissue samples with the same IgM+ IPCs with irregular nuclei suggests that the cells detected by immunofluorescence play a role in inflammation by recruiting CD15+ cells or by expressing CD15 themselves. However, Takei [13] found that anti-Leu M1 (antiCD15) antibody reacted against the surface antigen of a defined monoclonal B cell line (TKS-1) in the peripheral blood of patients with rheumatoid arthritis (RA), and that these cells produced IgM in the culture supernatant and had kappa light chain rearrangements in their DNA. They were also positive for Epstein-Barr nuclear antigen. Moreover, two EBV-positive B lymphoblastoid cell lines established using co-cultivated CD34+ cells isolated from the bone marrow of patients with RA and peripheral blood B lymphocytes from a healthy EBV-seronegative individual also expressed CD15 [14], thus indicating that EBV-infected B lymphoblastoid cells from patients with RA tend to acquire properties similar to those of $\mathrm{HL}$ cells [14].

The presence of IgM+B cells with irregular nuclei in the periglandial zone also suggests that the factors involved in cell recruitment are closely related to the characteristics of glandular cells. We estimate that these factors may be a result of lipid membrane changes. The production of IgM against the common membrane phospholipid, phosphatidylcholine (PC), is an important characteristic of peritoneal B1 cells [15]. These signify a sister B cell population that has the plasticity necessary to acquire the lymphocyte/monocyte characteristics typical of the IgM+ B cells with irregular nuclei described in this paper. A number of studies have investigated the role of PC in gastrointestinal damage and chronic inflammatory conditions such as UC: mucus from UC patients has a decreased PC content, and clinical trials have shown that the therapeutic addition of PC to the colonic mucus alleviates inflammatory activity [16].

The findings of this study indicate that B cells can show "lineage promiscuity" in inflammatory disease and that such cells correlate with the localization of CD15+ cells and a diagnosis of CD. In conclusion, these cells may play an intermediary role between innate immunity and adaptive immunity.

\section{References}

[1] T.W. LeBien, T.F. Tedder, B lymphocytes: how they develop and function, Blood 112 (2008) 1570-1580.

[2] M.A. Borrello, R.P. Phipps, The B/macrophage cell: an elusive link between CD5+ B lymphocytes and macrophages, Immunol. Today 17 (1996) 471-475.

[3] A.F. Popi, F.L. Motta, R.A. Mortara, S. Schenkman, J.D. Lopes, J.D. Lopes, M. Mariano, Co-ordinated expression of lymphoid and myeloid specific transcription factors during B-1b cell differentiation into mononuclear phagocytes in vitro, Immunology 126 (2009) 114-122.

[4] R. Kuppers, K. Rajewsky, M. Zhao, et al., Hodgkin disease: Hodgkin and Reed-Sternberg cells picked from histological sections show clonal immunoglobulin gene rearrangements and appear to be derived from B cells at various stages of development, Proc. Natl. Acad. Sci. U.S.A. 91 (1994) 10962-10966.

[5] M. Yurchenko, S.P. Sidorenko, Hodgkin's lymphoma: the role of cell surface receptors in regulation of tumor cell fate, Exp. Oncol. 32 (2010) 214-223.

[6] J.D. Lopes, M. Mariano, B-1 cell: the precursor of a novel mononuclear phagocyte with immuno-regulatory properties, Ann. Braz. Acad. Sci. 81 (2009) 489-496.

[7] M.A. Borrello, J. Palis, R.P. Phipps, The relationship of CD5+ B lymphocytes to macrophages: insights from normal biphenotypic B/macrophage cells, Int. Rev. Immunol. 20 (2001) 137-155.

[8] C. Defendenti, P. Sarzi-Puttini, S. Grosso, A.M. Croce, O. Senesi, S. Saibeni, S. Bollani, P.L. Almasio, S. Bruno, F. Atzeni, B lymphocyte intestinal homing in inflammatory bowel disease, BMC Immunol. 12 (2011) 71.

[9] W. Loveless, T. Feizi, M. Valeri, R. Day, S. Bay, A monoclonal antibody MIN/3/60, that recognizes the sulpho-Lewis(x) and sulpho-Lewis(a) sequences detects a sub-population of epithelial glycans in the crypts of human colonic epithelium, Hybridoma 20 (2001) 223-239.

[10] J.M. Munro, S.K. Lo, C. Corless, M.J. Robertson, N.C. Lee, R.L. Barnhill, D.S. Weinberg, M.P. Bevilacqua, Expression of sialyl-Lewis X, an E-selectin ligand, in inflammation, immune processes, and lymphoid tissues, Am. J. Pathol. 141 (1992) 6.

[11] P.D. Thomas, A. Forbes, A.B. Price, R.J. Nicholls, P.J. Ciclitira, Differential expression of cell adhesion molecules within inflamed ileal pouch mucosa: relationship to recruited cell subtypes, Eur. J. Gastroenterol. Hepatol. 14 (2002) 137-144.

[12] C. Capon, E. Maes, J.C. Michalski, H. Leffler, Y.S. Kim, Sd-antigen-like structures carried on core are prominent features of glycans from the mucin of normal human descending colon, Biochem. J. 358 (2001) 657-664.

[13] M. Takei, H. Kang, K. Tomura, E. Ikeda, M. Karasaki, H. Nakauchi, K. Okumura, S. Sawada, Expression of Leu M1 antigen on a monoclonal B cell line established from a patient with rheumatoid arthritis, Immunol. Lett. 23 (1989) 43-47.

[14] H. Inomata, M. Takei, H. Nakamura, S. Fujiwara, H. Shiraiwa, N. Kitamura, S Hirohata, H. Masuda, J. Takeuchi, S. Sawada, Epstein-Barr-virus-infected CD15 (Lewis X)-positive Hodgkin-lymphoma-like B cells in patients with rheumatoid arthritis, Open Rheumatol. J. 3 (2009) 41-47.

[15] T.J. Mercolino, A.L. Loke, A. Afshari, D. Sasser, W.W. Travis, L.W. Arnold, G. Haughton, Restricted immunoglobulin variable region gene usage by normal Ly-1 (CD5+) B cells that recognize phosphatidyl choline, J. Exp. Med. 169 (1989) $1869-1877$.

[16] H. Schneider, A. Braun, Füllekrug, W. Stremmel, R. Ehehalt, Lipid based therapy for ulcerative colitis. Modulation of intestinal mucus membrane phospholipids as a tool to influence inflammation, Int. J. Mol. Sci. 11 (2010) 4149-4164. 